\title{
A NOTE ON RELATIVE PSEUDOCOMPACTNESS IN THE CATEGORY OF FRAMES
}

\author{
THEMBA DUBE
}

(Received 15 February 2012)

\begin{abstract}
A subspace $S$ of Tychonoff space $X$ is relatively pseudocompact in $X$ if every $f \in C(X)$ is bounded on $S$. As is well known, this property is characterisable in terms of the functor $v$ which reflects Tychonoff spaces onto the realcompact ones. A device which exists in the category CRegFrm of completely regular frames which has no counterpart in Tych is the functor which coreflects completely regular frames onto the Lindelöf ones. In this paper we use this functor to characterise relative pseudocompactness.
\end{abstract}

2010 Mathematics subject classification: primary 06D22; secondary 06E20, 54C40.

Keywords and phrases: frame, frame homomorphism, real-valued continuous functions on a frame, Lindelöf coreflection, relative pseudocompactness.

\section{Introduction}

A subspace $S$ of Tychonoff space $X$ is relatively pseudocompact in $X$ if every $f \in C(X)$ is bounded on $S$. As is well known, this property is characterisable in terms of the functor $v$ which reflects Tychonoff spaces onto the realcompact ones. A device which exists in the category CRegFrm of completely regular frames which has no counterpart in Tych is the functor which coreflects completely regular frames onto the Lindelöf ones. In this paper we use this functor to characterise relative pseudocompactness.

For ease of reference we reproduce from [8] the topological proposition which we shall extend to the category CRegFrm, using the functor $\lambda$, which coreflects completely regular frames to the Lindelöf ones, instead of the sometimes recalcitrant $v$. Proposition 1.1 (Blair and Swardson [8]). The following are equivalent for $a$ subspace $S$ of a Tychonoff space $X$ :

(a) $S$ is relatively pseudocompact in $X$;

(b) $\mathrm{cl}_{v X} S$ is compact;

(c) $\operatorname{cl}_{\beta X} S \subseteq v X$.

The author acknowledges financial support from the National Research Foundation of South Africa under the grant IFR2011040100021.

(C) 2012 Australian Mathematical Publishing Association Inc. 0004-9727/2012 \$16.00 
The frame version of the equivalence of statements (1) and (2) is shown in [10] in terms of the functor $v$. In this note we obtain characterisations of relative pseudocompactness in CRegFrm in terms of the functor $\lambda$. In our context, statement (c) will be couched in the language of nuclei.

The paper is organised as follows. In Section 2 we fix notation and recall a few facts we shall need, such as the construction of the coreflections $v L$ and $\lambda L$. Our general reference for frames is the recent book of Picado and Pultr [16]. The main result is in Section 3, where we also observe another topological result the frame analogue of which is improved in CRegFrm by the functor $\lambda$. The result in question is the following (see [13, 8.10(a) and 8.10(b)]): if $S$ is a $C$-embedded subspace of $X$, then $\mathrm{cl}_{v S}=v S$; with a partial converse if $X$ or $v X$ is normal. Now in the frame version normality comes for free because the frame $\lambda L$ is normal. We shall thus have a full converse (Proposition 3.7).

\section{Assembling the requisite tools}

2.1. Fixing notation. All our frames are completely regular, and we denote the category they form by CRegFrm. For a detailed discussion on the ring of real-valued functions on a frame, the reader is encouraged to consult $[2,3]$. We denote the top element and the bottom element of a frame $L$ by $1_{L}$ and $0_{L}$, respectively, dropping the subscript if $L$ is clear from the context. By a quotient map we mean a surjective frame homomorphism. If $h: L \rightarrow M$ is a quotient map, we shall also say $M$ is a quotient of $L$. Given a frame $L$, by a closed quotient of $L$ we mean any frame of the form $\uparrow a$, for $a \in L$. In this case the unmentioned quotient map will always be the frame homomorphism

$$
\kappa_{a}: L \rightarrow \uparrow a \text { given by } x \mapsto a \vee x .
$$

An open quotient of $L$ is a frame of the form $\downarrow a$, for $a \in L$, with the quotient map

$$
v_{a}: L \rightarrow \downarrow a \text { given by } x \mapsto a \wedge x \text {. }
$$

A frame homomorphism is called dense if it maps only the bottom element to the bottom element. We denote, as usual, the right adjoint of a homomorphism $h: L \rightarrow M$ by $h_{*}$, and recall that $h$ is onto if and only if $h h_{*}=\mathrm{id}_{M}$. If $h$ is a dense quotient map, then $h\left(a^{*}\right)=h(a)^{*}$ and $h_{*}\left(b^{*}\right)=\left(h_{*}(b)\right)^{*}$ for all $a \in L$ and $b \in M$.

An element $p$ of a frame is called a point if $p \neq 1$ and $a \wedge b \leq p$ implies $a \leq p$ or $b \leq p$. We denote by $\operatorname{Pt}(L)$ the set of all points of $L$. The points of a regular frame are precisely those elements which are maximal strictly below the top.

As in [3], we denote by $\mathcal{R} L$ the ring of all real-valued continuous functions on $L$. The reader will recall that the underlying set of this ring is the set of all frame homomorphisms $\mathfrak{L}(\mathbb{R}) \rightarrow L$, where $\mathfrak{Q}(\mathbb{R})$ denotes the frame of reals. A cozero element of $L$ is an element of the form $\varphi((-, 0) \vee(0,-))$, for some $\varphi \in \mathcal{R} L$. An element $a$ of $L$ is a cozero element if and only if there is a sequence $\left(a_{n}\right)$ in $L$ such that $a_{n} \ll a$ for each $n$ and $a=\bigvee a_{n}$. The set of all cozero elements of $L$ is called the cozero part of $L$ 
and is denoted by $\operatorname{Coz} L$. It is a sub- $\sigma$-frame of $L$ which generates $L$ by joins precisely when $L$ is completely regular. General properties of cozero elements and cozero parts of frames can be found in [5].

A function $\alpha \in \mathcal{R} L$ is bounded if $\alpha(p, q)=1_{L}$ for some $p$ and $q$ in $\mathbb{Q}$, and $L$ is said to be pseudocompact if every element of $\mathcal{R} L$ is bounded. We rephrase a characterisation of pseudocompact frames from [5] that we shall use in terms of what Ball and WaltersWayland [2] call towers. We shall slightly modify the terminology from [2]. A tower in a frame $L$ is a sequence $\left(a_{n}\right)$, indexed by $\mathbb{N}$, of elements of $L$ such that $a_{n} \leq a_{n+1}$ for every $n$, and $\bigvee a_{n}=1$. A tower $\left(a_{n}\right)$ terminates if $a_{n}=1$ for some index $n$. A cozero tower is a tower consisting of cozero elements. A cozero tower $\left(c_{n}\right)$ is regular if $c_{n} \ll c_{n+1}$ for each $n$. Since $a \ll b$ in $L$ implies $a \ll c \ll b$ for some $c \in \operatorname{Coz} L$, we have that

$L$ is pseudocompact if and only if every regular cozero tower in $L$ terminates.

2.2. The coreflections $\boldsymbol{\beta} \boldsymbol{L}, \lambda \boldsymbol{L}$ and $\boldsymbol{v} \boldsymbol{L}$. The compact, completely regular coreflection of any completely regular frame $L$ (the frame counterpart of the StoneČech compactification of Tychonoff spaces), denoted $\beta L$, was first constructed by Banaschewski and Mulvey [7] as the frame of completely regular ideals of $L$. It can also be realised as the frame of regular ideals of $\operatorname{Coz} L$ (see, for instance, [6]). For our purposes it is convenient to adopt this latter view. We denote the right adjoint of the join map $j_{L}: \beta L \rightarrow L$ by $r_{L}$, and recall that

$$
r_{L}(a)=\{c \in \operatorname{Coz} L \mid c \ll a\} .
$$

We remind the reader that if $L$ is normal, then $r_{L}$ preserves finite joins (see [1]).

Madden and Vermeer [14] have shown that regular Lindelöf frames are coreflective in CRegFrm. We recall the construction of the coreflection. An ideal of $\operatorname{Coz} L$ is a $\sigma$-ideal if it is closed under countable joins. The regular Lindelöf coreflection of $L$, denoted $\lambda L$, is the frame of $\sigma$-ideals of $\operatorname{Coz} L$. The join map $\lambda_{L}: \lambda L \rightarrow L$ is a dense onto frame homomorphism, and is the attendant coreflection map. We denote by $k_{L}$ the dense onto frame homomorphism

$$
k_{L}: \beta L \rightarrow \lambda L \quad \text { given by } k_{L}(I)=\langle I\rangle_{\sigma},
$$

where $\langle\cdot\rangle_{\sigma}$ signifies $\sigma$-ideal generation in $\operatorname{Coz} L$. It is not too difficult to show that $j_{L}=\lambda_{L} \cdot k_{L}$, and that $k_{L}: \beta L \rightarrow \lambda L$ is (isomorphic to) the Stone-Čech compactification of $\lambda L$.

Realcompact frames are coreflective in CRegFrm (see, for instance, [6, 15] for details). The realcompact coreflection of $L$, denoted $v L$, is constructed in the following manner. For any $a \in L$, let $[a]=\{x \in \operatorname{Coz} L \mid x \leq a\}$. Note that if $a \in \operatorname{Coz} L$, then $[a]$ is the principal ideal of $\operatorname{Coz} L$ generated by $a$. The map $\ell: \lambda L \rightarrow \lambda L$ given by

$$
\ell(J)=[\bigvee J] \wedge \bigwedge\{P \in \operatorname{Pt}(\lambda L) \mid J \leq P\}
$$


is a nucleus. The frame $v L$ is defined to be $\operatorname{Fix}(\ell)$. We denote by $\ell_{L}$ the dense onto frame homomorphism $\lambda L \rightarrow v L$ effected by $\ell$. The join map $v_{L}: v L \rightarrow L$ is a dense onto frame homomorphism. For any $a \in L$,

$$
\left(\lambda_{L}\right)_{*}(a)=\left(v_{L}\right)_{*}(a)=[a] .
$$

The frames $\lambda L$ and $v L$ have identical cozero parts, namely,

$$
\operatorname{Coz}(\lambda L)=\operatorname{Coz}(v L)=\{[c] \mid c \in \operatorname{Coz} L\}
$$

2.3. Coz-onto and $\boldsymbol{C}$-quotient maps. A frame homomorphism $h: L \rightarrow M$ is cozonto if for every $b \in \operatorname{Coz} M$ there is an $a \in \operatorname{Coz} L$ such that $h(a)=b$. It is a $C$-quotient map if it is a quotient map and for every $\alpha \in \mathcal{R} M$ there is an $\hat{\alpha} \in \mathcal{R} L$ such that the triangle

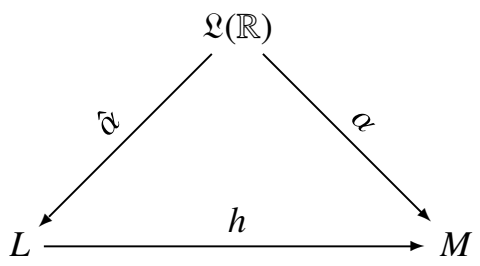

commutes. If $L$ is normal and $a \in L$, then $\kappa_{a}: L \rightarrow \uparrow a$ is coz-onto (in fact, it is a $C$-quotient map [2, Theorem 8.3.3]) and, hence,

$$
\operatorname{Coz}(\uparrow a)=\{a \vee c \mid c \in \operatorname{Coz} L\}
$$

\section{The results}

In [10], relative pseudocompactness for frames is defined analogously to spaces. We recall the definition which, incidentally, is 'conservative' in the sense that $S$ is

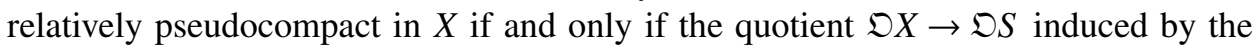
subspace embedding $S \hookrightarrow X$ is relatively pseudocompact in $\mathfrak{D} X$.

Definition 3.1. A quotient $h: L \rightarrow M$ of $L$ is relatively pseudocompact (in $L$ ) if, for every homomorphism $f: \mathfrak{L}(\mathbb{R}) \rightarrow L$, the composite $h f$ is bounded.

We shall need the following lemma which we believe is folklore. Because we do not have a reference for it, we shall provide a proof. Recall that if $h: L \rightarrow M$ is a dense homomorphism, then $h_{*} h(a) \leq b$ whenever $a<b$ in $L$. For a cover $C$ of a regular frame $L$, denote by $\check{C}$ the cover

$$
\check{C}=\{x \in L \mid x<c \text { for some } c \in C\} .
$$

Lemma 3.2. If $h: L \rightarrow M$ is a dense homomorphism with $M$ compact and $L$ regular, then $L$ is compact. 
Proof. Let $C$ be a cover of $L$. Then $h[\check{C}]$ is a cover of $M$ and, hence, by compactness, there are finitely many elements $x_{1}, \ldots, x_{n}$ in $\check{C}$ such that

$$
h\left(x_{1}\right) \vee \cdots \vee h\left(x_{n}\right)=1 \text {. }
$$

For each $i=1, \ldots, n$, pick $c_{i} \in C$ with $x_{i} \prec c_{i}$. Since $x_{1} \vee \cdots \vee x_{n} \prec c_{1} \vee \cdots \vee c_{n}$ and $h$ is dense,

$$
1=h_{*} h\left(x_{1} \vee \cdots \vee x_{n}\right) \leq c_{1} \vee \cdots \vee c_{n} .
$$

Therefore, $L$ is compact.

Next, observe that if $\phi: A \rightarrow B$ is a frame homomorphism, then for any $a \in L$ the map $\phi_{a}: \uparrow a \rightarrow \uparrow \phi(a)$, mapping as $\phi$, is a frame homomorphism making the following diagram commute.

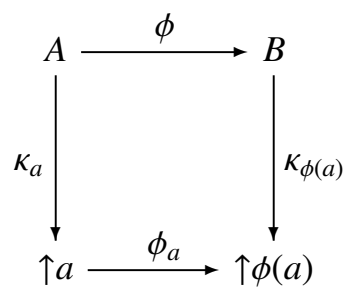

In such a case we shall say that $\phi_{a}$ is the homomorphism induced by a from $\phi$.

Given a frame $L$ and $a \in L$, the notation $\uparrow[a]$ is ambiguous because the element $[a]$ resides both in $\lambda L$ and $v L$. Let us agree that if we write $\uparrow[a]$ we shall be meaning the closed quotient of $\lambda L$ determined by [a]. If $h: L \rightarrow M$ is a homomorphism, we shall abbreviate the closed quotient $\uparrow\left(h v_{L}\right)_{*}(0)$ of $v L$ as $\uparrow(h v)_{*}(0)$ or $\uparrow v_{*} h_{*}(0)$; and similarly for $\lambda L$. Thus, $\uparrow\left[h_{*}(0)\right]=\uparrow(h \lambda)_{*}(0)$.

We remind the reader that nuclei on a frame are compared pointwise. That is, if $j$ and $k$ are nuclei on $L$, then $j \leq k$ means $j(x) \leq k(x)$ for every $x \in L$. We denote the closed nucleus $a \vee(\cdot)$ by $c_{a}$. In the proof of the following result we shall need to know how the right adjoint of $k_{L}: \beta L \rightarrow \lambda L$ is calculated. It is shown in [11] that, for any $I \in \lambda L$,

$$
\left(k_{L}\right)_{*}(I)=\bigvee_{\beta L}\left\{r_{L}(a) \mid a \in I\right\} .
$$

Proposition 3.3. Let $h: L \rightarrow M$ be a quotient of $L$. The following statements are equivalent:

(1) $M$ is relatively pseudocompact in $L$;

(2) $\uparrow(h v)_{*}(0)$ is compact;

(3) $\uparrow(h \lambda)_{*}(0)$ is compact;

(4) $\left(k_{L}\right)_{*} k_{L} \leq \mathfrak{c}_{r_{L}\left(h_{*}(0)\right)}$.

Proof. That (1) and (2) are equivalent is shown in [10, Proposition 3.2].

$(2) \Rightarrow(3)$ : Assume that (2) holds. To prove (3), it suffices, by Lemma 3.2, to produce a dense homomorphism $\uparrow(h \lambda)_{*}(0) \rightarrow \uparrow(h v)_{*}(0)$. Since $(h \lambda)_{*}(0)=\lambda_{*} h_{*}(0)=$ $\left[h_{*}(0)\right]$, we have $(h v)_{*}(0)=\ell_{L}\left((h \lambda)_{*}(0)\right)$, and so we may define

$$
\varphi: \uparrow(h \lambda)_{*}(0) \rightarrow \uparrow(h v)_{*}(0)
$$


to be the homomorphism induced by $(h \lambda)_{*}(0)$ from the homomorphism $\ell_{L}: \lambda L \rightarrow v L$ as per diagram ( $\dagger$ ) above. We show that $\varphi$ is dense by showing that the only cozero element it sends to the bottom is the bottom, which will prove the result by complete regularity. We will denote join in $v L$ by $\sqcup$. Since $\lambda L$ is normal,

$$
\operatorname{Coz}\left(\uparrow(h \lambda)_{*}(0)\right)=\left\{[c] \vee\left[h_{*}(0)\right] \mid c \in \operatorname{Coz} L\right\} .
$$

Consider any $c \in \operatorname{Coz} L$ for which $\varphi\left([c] \vee\left[h_{*}(0)\right]\right)=(h v)_{*}(0)$. This implies

$$
\ell_{L}\left([c] \vee\left[h_{*}(0)\right]\right)=\left[h_{*}(0)\right],
$$

so that

$$
[c] \sqcup\left[h_{*}(0)\right]=\left[h_{*}(0)\right],
$$

whence $[c] \leq\left[h_{*}(0)\right]$. Thus, $[c] \vee\left[h_{*}(0)\right]=\left[h_{*}(0)\right]$. Therefore, $\varphi$ is dense and, hence, $\uparrow(h \lambda)_{*}(0)$ is compact.

$(3) \Rightarrow(1)$ : The proof we give is adapted from that of the implication $(\Leftarrow)$ in $[10$, Proposition 3.2]. Let $f: \mathfrak{L}(\mathbb{R}) \rightarrow L$ be a frame homomorphism. Since $\mathfrak{L}(\mathbb{R})$ is Lindelöf, there is a frame homomorphism $\tilde{f}: \mathfrak{Q}(\mathbb{R}) \rightarrow \lambda L$ such that the triangle

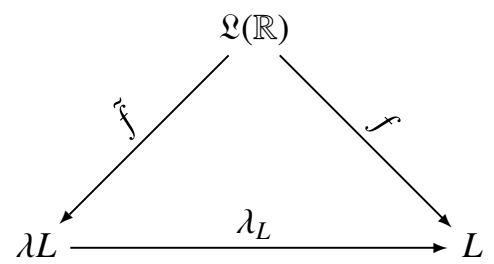

commutes. Since $\{(p, q) \mid p, q \in \mathbb{Q}\}$ is a cover of $\mathfrak{Q}(\mathbb{R})$, the set

$$
\left\{\tilde{f}(p, q) \vee(h \lambda)_{*}(0) \mid p, q \in \mathbb{Q}\right\}
$$

is a (directed) cover of the compact frame $\uparrow(h \lambda)_{*}(0)$ and, hence, for some $s, t \in \mathbb{Q}$,

$$
\tilde{f}(s, t) \vee(h \lambda)_{*}(0)=1_{\lambda L} .
$$

Applying the map $h \lambda_{L}$ to this, and taking into cognisance that $\lambda_{L} \tilde{f}=f$, we obtain $h f(s, t)=1_{M}$, which shows that $h f$ is bounded.

$(3) \Rightarrow(4)$ : Let $I \in \beta L$, and consider any $J \in \beta L$ with $J \prec\left(k_{L}\right)_{*} k_{L}(I)$. Then

$$
J^{*} \vee \bigvee_{\beta L}\left\{r_{L}(a) \mid a \in k_{L}(I)\right\}=1_{\beta L}
$$

By the compactness of $\beta L$, there is an $a \in k_{L}(I)$ such that $J^{*} \vee r_{L}(a)=1_{\beta L}$. Since $j_{L}=\lambda_{L} \cdot k_{L}$, so that $r_{L}=\left(k_{L}\right)_{*}\left(\lambda_{L}\right)_{*}$, on applying the homomorphism $k_{L}$ to the previous equality we get

$$
k_{L}\left(J^{*}\right) \vee[a]=1_{\lambda L}
$$


Because $a \in k_{L}(I)$, there is a sequence $\left(a_{n}\right)$ in $I$ such that $a \leq \bigvee a_{n}$. Consequently,

$$
k_{L}\left(J^{*}\right) \vee \bigvee_{n=1}^{\infty}\left[a_{n}\right]=1_{\lambda L}
$$

so that the set

$$
\left\{\left[h_{*}(0)\right] \vee k_{L}\left(J^{*}\right) \vee\left[a_{n}\right] \mid n \in \mathbb{N}\right\}
$$

is a cover of the frame $\uparrow\left[h_{*}(0)\right]$. Thus, by compactness of this frame, there is a $b \in I$ such that

$$
k_{L}\left(J^{*}\right) \vee\left[h_{*}(0)\right] \vee[b]=1_{\lambda L} .
$$

Since $\lambda L$ is normal, there is a $c \in \operatorname{Coz} L$ such that

$$
[c] \leq k_{L}(J)^{*} \quad \text { and } \quad[c] \vee\left[h_{*}(0)\right] \vee[b]=1_{\lambda L} .
$$

Now, since $k_{L}: \beta L \rightarrow \lambda L$ is the Stone-Čech compactification of the normal frame $\lambda L$, $\left(k_{L}\right)_{*}$ preserves finite joins. Thus, in light of the equality $\left(k_{L}\right)_{*}\left(\lambda_{L}\right)_{*}=r_{L}$,

$$
r_{L}(c) \vee r_{L}\left(h_{*}(0)\right) \vee r_{L}(b)=r_{L}(c) \vee\left(r_{L}\left(h_{*}(0)\right) \vee r_{L}(b)\right)=1_{\beta L},
$$

which implies

$$
r_{L}\left(c^{*}\right) \leq r_{L}\left(h_{*}(0)\right) \vee I=\mathfrak{c}_{r_{L}\left(h_{*}(0)\right)}(I) .
$$

Since $[c]^{*}=\left[c^{*}\right]$ and $k_{L}$ preserves pseudocomplements, the inequality $[c] \leq k_{L}\left(J^{*}\right)$ implies $k_{L}\left(J^{* *}\right) \leq\left[c^{*}\right]$, so that

$$
J \leq J^{* *} \leq\left(k_{L}\right)_{*}\left(\left[c^{*}\right]\right)=r_{L}\left(c^{*}\right) \leq \mathfrak{c}_{r_{L}\left(h_{*}(0)\right)}(I) .
$$

It follows therefore that $\left(k_{L}\right)_{*} k_{L} \leq \mathfrak{c}_{r_{L}\left(h_{*}(0)\right)}$ because $\left(k_{L}\right)_{*}\left(k_{L}\right)(I)$ is the join of elements which are rather below it.

(4) $\Rightarrow(3)$ : Since $\uparrow\left[h_{*}(0)\right]$ is Lindelöf, to show that it is compact it suffices to show that it is pseudocompact. So let $\left(J_{n}\right)$ be a sequence of cozero elements of $\uparrow\left[h_{*}(0)\right]$ such that

$$
J_{1} \ll J_{2} \ll \cdots \quad \text { and } \bigvee J_{n}=1_{\lambda L} .
$$

Since $\lambda L$ is normal, the homomorphism $\kappa_{\left[h_{*}(0)\right]}: \lambda L \rightarrow \uparrow\left[h_{*}(0)\right]$ is a $C$-quotient map [2, Theorem 8.3.3]. Therefore, by [2, Theorem 7.2.7], there is a sequence $\left(U_{n}\right)$ in $\operatorname{Coz}(\lambda L)$ such that

$$
\left[h_{*}(0)\right] \vee U_{n} \leq J_{n}, \quad U_{n} \ll U_{n+1} \text { for every } n \quad \text { and } \bigvee_{\lambda L} U_{n}=1_{\lambda L} .
$$

For each $n$, pick $u_{n} \in \operatorname{Coz} L$ such that $U_{n}=\left[u_{n}\right]$, and define an element $I$ of $\beta L$ by

$$
I=\bigvee_{\beta L}\left\{r_{L}\left(u_{n}\right) \mid n=1,2, \ldots\right\}
$$


Then

$$
\begin{aligned}
k_{L}(I) & =\bigvee_{\lambda L}\left\{k_{L} r_{L}\left(u_{n}\right) \mid n=1,2, \ldots\right\} \\
& =\bigvee_{\lambda L}\left\{\left[u_{n}\right] \mid n=1,2, \ldots\right\} \\
& =1_{\lambda L},
\end{aligned}
$$

which implies $\left(k_{L}\right)_{*} k_{L}(I)=1_{\beta L}$ and, hence, by hypothesis,

$$
r_{L}\left(h_{*}(0)\right) \vee \bigvee_{\beta L}\left\{r_{L}\left(u_{n}\right) \mid n=1,2, \ldots\right\}=1_{\beta L}
$$

By compactness of $\beta L$, there is an index $n$ such that $r_{L}\left(h_{*}(0)\right) \vee r_{L}\left(u_{n}\right)=1_{\beta L}$. Applying the map $k_{L}$, we obtain $\left[h_{*}(0)\right] \vee\left[u_{n}\right]=1_{\lambda L}$, whence $J_{n}=1_{\lambda L}$. Therefore, $\uparrow\left[h_{*}(0)\right]$ is pseudocompact and, hence, compact.

REMARK 3.4. In spite of our predilection for all things frame-theoretic, we should concede that the equivalence in statement (4) is not transparent. In localic terms it says precisely what the corresponding topological one says; to wit, for a sublocale $S$ of a locale $X, \mathrm{cl}_{\beta X} S \leq v X$, where the comparison is contemplated in the lattice of sublocales of $\beta X$.

REMARK 3.5. In proving that the map $\varphi: \uparrow(h \lambda)_{*}(0) \rightarrow \uparrow(h v)_{*}(0)$ in the implication $(2) \Rightarrow(3)$ is dense, we could not simply have counted on the fact that $\ell_{L}: \lambda L \rightarrow v L$ is dense. Here is an example to see why. Let $L$ be a non-Boolean frame and b: $L \rightarrow \mathfrak{B} L$ be the Booleanisation map $x \mapsto x^{* *}$. Let $a$ be an element of $L$ for which $a \neq a^{* *}$. Then the map $b_{a}: \uparrow a \rightarrow \uparrow a^{* *}$ induced by $a$ from $b$ is not dense. Indeed, $a^{* *}$ is a nonzero element of $\uparrow a$ mapped to the zero of $\uparrow a^{* *}$ by $b_{a}$.

In the case of open quotients $L \rightarrow \downarrow a$, the characterisation above can be expressed solely in terms of the elements of the frame $L$ without mention of the Lindelöf coreflection. To prove that we will need to take note of the following facts.

(a) Recall that an element of a frame is dense if its pseudocomplement is zero. If $a<b$ and $a$ is dense, then $b=1$.

(b) If $a \in L$, then $a \vee a^{*}$ is dense in the frame $\uparrow a^{*}$. Indeed, by [12, Lemma 4.5], the pseudocomplement of $a \vee a^{*}$ in $\uparrow a^{*}$ is $\left(\left(a \vee a^{*}\right) \wedge a^{* *}\right)^{*}=a^{*}$, the bottom element of $\uparrow a^{*}$.

Corollary 3.6. For any $a \in L$, the open quotient $v_{a}: L \rightarrow \downarrow a$ is relatively pseudocompact in $L$ if and only if for every cozero tower $\left(c_{n}\right)$ in $L$, there is an index $m$ such that $a \leq c_{m}$.

Proof. $(\Rightarrow)$ Assume that $\downarrow a$ is relatively pseudocompact in $L$, and let $\left(c_{n}\right)$ be a cozero tower in $L$. Since $\left(v_{a}\right)_{*}(0)=a^{*}$, Proposition 3.3 implies that $\uparrow\left[a^{*}\right]$ is compact. Since 
the $c_{n}$ are cozero elements, we have that

$$
\bigvee_{\lambda L}\left\{\left[c_{n}\right] \mid n \in \mathbb{N}\right\}=1_{\lambda L}
$$

and hence the set $\left\{\left[a^{*}\right] \vee\left[c_{n}\right] \mid n \in \mathbb{N}\right\}$ is a cover of $\uparrow\left[a^{*}\right]$. By compactness of this frame, there is an index $m$ such that $\left[a^{*}\right] \vee\left[c_{m}\right]=1_{\lambda L}$, which implies $a^{*} \vee c_{m}=1$, whence $a \leq c_{m}$.

$(\Leftarrow)$ We show that $\uparrow\left[a^{*}\right]$ is compact. Because this frame is Lindelöf, it is enough to show that it is pseudocompact. Write $\kappa$ for the closed quotient map $\kappa_{\left[a^{*}\right]}: \lambda L \rightarrow \uparrow\left[a^{*}\right]$. Consider any regular cozero tower

$$
\left[a^{*}\right] \vee\left[c_{1}\right] \ll\left[a^{*}\right] \vee\left[c_{2}\right] \ll \cdots
$$

in $\uparrow\left[a^{*}\right]$. Since $\lambda L$ is normal, $\kappa: \lambda L \rightarrow \uparrow\left[a^{*}\right]$ is a $C$-quotient map, and so there is a regular cozero tower $\left(\left[d_{n}\right]\right)$ in $\lambda L$ such that

$$
\kappa\left(\left[d_{n}\right]\right) \leq\left[a^{*}\right] \vee\left[c_{n}\right] \quad \text { for every } n .
$$

Now the sequence $\left(d_{n}\right)$ is a cozero tower in $L$, so, by the present hypothesis, there is an index $m$ such that $a \leq d_{m}$. Consequently,

$$
\kappa([a]) \leq \kappa\left(\left[d_{m}\right]\right) \leq\left[a^{*}\right] \vee\left[c_{m}\right] \ll\left[a^{*}\right] \vee\left[c_{m+1}\right] .
$$

But $\kappa([a])=\left[a^{*}\right] \vee[a]=[a]^{*} \vee[a]$, so that it is a dense element in $\uparrow\left[a^{*}\right]$, whence $\left[a^{*}\right] \vee\left[c_{m+1}\right]=1_{\lambda L}$, implying $\uparrow\left[a^{*}\right]$ is pseudocompact, and hence compact. Therefore, $\downarrow a$ is relatively pseudocompact in $L$.

We now prove the result alluded to at the end of the Introduction.

Proposition 3.7. Let $h: L \rightarrow$ M be a quotient map, and $g: \uparrow(h \lambda)_{*}(0) \rightarrow$ M be the frame homomorphism mapping as $h \lambda$. Then $g: \uparrow(h \lambda)_{*}(0) \rightarrow M$ is the Lindelöf coreflection of $M$ if and only if $h$ is a $C$-quotient map.

Proof. $(\Rightarrow)$ We show that $h$ is coz-onto and almost coz-codense, which will establish the implication by [2, Theorem 7.2.3]. Let $c \in \operatorname{Coz} M$. Then there is a $J \in$ $\operatorname{Coz}\left(\uparrow(h \lambda)_{*}(0)\right)$ such that $g(J)=c$. That is, there is a $d \in \operatorname{Coz} L$ such that

$$
c=h \lambda\left(\left[h_{*}(0)\right] \vee[d]\right)=h\left(h_{*}(0)\right) \vee h(d)=h(d) .
$$

Therefore, $h$ is coz-onto.

Next, suppose that $h(c)=1$ for some $c \in \operatorname{Coz} L$. Then $\left[h_{*}(0)\right] \vee[c]$ is a cozero element of $\uparrow(h \lambda)_{*}(0)$ with

$$
g\left(\left[h_{*}(0)\right] \vee[c]\right)=h(c)=1 .
$$

So $\left[h_{*}(0)\right] \vee[c]=1_{\lambda L}$ since the Lindelöf coreflection is always coz-codense. By normality of $\lambda L$, there is a $d \in \operatorname{Coz} L$ such that

$$
[d] \leq\left[h_{*}(0)\right] \text { and }[d] \vee[c]=1_{\lambda L} .
$$


But this implies $d \vee c=1$ and $d \leq h_{*}(0)$, and the latter implies $h(d)=0$. Therefore, $h$ is almost coz-codense.

$(\Leftarrow)$ Since $\uparrow(h \lambda)_{*}(0)$ is Lindelöf and $g$ is dense onto, $g: \uparrow(h \lambda)_{*}(0) \rightarrow M$ is a Lindelöfication of $M$. By [2, Corollary 8.2.13], it suffices to show that $g$ is coz-onto and coz-codense. That can be done along the same lines as in the first implication.

By way of concluding, we say a word about relative pseudocompactness of lifted quotients. Let us explain what we mean by 'lifted quotients'. For every homomorphism $h: L \rightarrow M$ there is a homomorphism $h^{\lambda}: \lambda L \rightarrow \lambda M$ such that the square on the right in the diagram ( $\ddagger$ ) below commutes. We call $h^{\lambda}$ the $\lambda$-lift of $h$. Similarly, there is an $v$-lift which makes the corresponding square commute, that is, for which $h \cdot v_{L}=v_{M} \cdot h^{v}$.

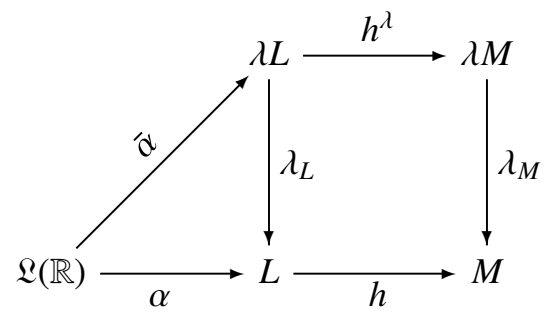

Neither the $\lambda$-lift nor the $v$-lift need be a quotient map if $h$ is a quotient map. It is shown in [9, Lemma 2.3] that $h^{\lambda}$ is a quotient map precisely when $h$ is coz-onto. In fact, if $h$ is coz-onto, then $h^{v}: v L \rightarrow v M$ is a quotient map. Indeed, for any $c \in \operatorname{Coz} M$, take $d \in \operatorname{Coz} L$ such that $h(d)=c$. Since $h^{v}=\ell_{M} \cdot h^{\lambda} \cdot\left(\ell_{L}\right)_{*}$ and $(\ell)_{*}$ is the inclusion $v L \hookrightarrow \lambda L$, it is easy to see that $h^{v}([d])=[c]$, so that $h^{v}$ maps onto $\operatorname{Coz}(v M)$, and hence onto $v M$, by complete regularity. In our last proposition we shall thus impose the condition that $h$ be coz-onto.

Proposition 3.8. Let $h: L \rightarrow M$ be a coz-onto homomorphism. Then the following statements are equivalent:

(1) $h: L \rightarrow M$ is relatively pseudocompact;

(2) $h^{\lambda}: \lambda L \rightarrow \lambda M$ is relatively pseudocompact;

(3) $h^{v}: v L \rightarrow v M$ is relatively pseudocompact.

Proof. $(1) \Rightarrow(2)$ : Let $f: \mathfrak{L}(\mathbb{R}) \rightarrow \lambda L$ be a frame homomorphism. By hypothesis, there are elements $p, q \in \mathbb{Q}$ such that $h\left(\lambda_{L} f\right)(p, q)=1_{M}$. Since $h \lambda_{L}=\lambda_{M} h^{\lambda}$, this implies $\lambda_{M}\left(h^{\lambda} f(p, q)\right)=1_{M}$. Since $h^{\lambda} f(p, q) \in \operatorname{Coz}(\lambda M)$ and $\lambda_{M}$ is coz-codense, it follows that $h^{\lambda} f(p, q)=1_{\lambda M}$, showing that $h^{\lambda}: \lambda L \rightarrow \lambda M$ is relatively pseudocompact.

$(2) \Rightarrow(1)$ : Let $\alpha: \mathfrak{L}(\mathbb{R}) \rightarrow L$ be a frame homomorphism. Since $\mathfrak{Q}(\mathbb{R})$ is Lindelöf, there is a homomorphism $\bar{\alpha}: \mathfrak{L}(\mathbb{R}) \rightarrow \lambda L$ such that the triangle on the left of the diagram ( $\ddagger)$ above commutes. Then

$$
h \alpha=\left(h \lambda_{L}\right) \bar{\alpha}=\left(\lambda_{M} h^{\lambda}\right) \bar{\alpha}
$$


By the current hypothesis, $\lambda_{M} h^{\lambda}$ is bounded, and therefore the composite $\lambda_{M} h^{\lambda} \bar{\alpha}$ is bounded, that is, $h \alpha$ is bounded, as required.

The equivalence of (1) and (3) can be shown similarly, using, for the implication $(3) \Rightarrow(1)$, that $\mathfrak{L}(\mathbb{R})$ is realcompact.

\section{References}

[1] D. Baboolal and B. Banaschewski, 'Compactification and local connectedness of frames', J. Pure Appl. Algebra 70 (1991), 3-16.

[2] R. N. Ball and J. Walters-Wayland, ' $C$ - and $C^{*}$-quotients in pointfree topology', Dissertationes Math. (Rozprawy Mat.) 412 (2002), 62.

[3] B. Banaschewski, The Real Numbers in Pointfree Topology, Textos de Matemática Série B, 12 (Departamento de Matemática da Universidade de Coimbra, Coimbra, 1997).

[4] B. Banaschewski and C. Gilmour, 'Stone-Čech compactification and dimension theory for regular $\sigma$-frames', J. Lond. Math. Soc. 2(127) (1989), 1-8.

[5] B. Banaschewski and C. Gilmour, 'Pseudocompactness and the cozero part of a frame', Comment. Math. Univ. Carolin. 37(3) (1996), 577-587.

[6] B. Banaschewski and C. Gilmour, 'Realcompactness and the cozero part of a frame', Appl. Categ. Structures 9 (2001), 395-417.

[7] B. Banaschewski and C. Mulvey, 'Stone-Čech compactification of locales. I', Houston J. Math. 6 (1980), 301-312.

[8] R. L. Blair and M. A. Swardson, 'Spaces with an Oz Stone-Čech compactification', Topology Appl. 36 (1990), 73-92.

[9] T. Dube, 'Some notes on $C$ - and $C^{*}$-quotients of frames', Order 25 (2008), 369-375.

[10] T. Dube and P. Matutu, 'A few points on pointfree pseudocompactness', Quaest. Math. 30 (2007), 451-464.

[11] T. Dube and I. Naidoo, 'Round squares in the category of frames', Houston J. Math., to appear.

[12] T. Dube and J. Walters-Wayland, 'Coz-onto frame maps and some applications', Appl. Categ. Structures 15 (2007), 119-133.

[13] L. Gillman and M. Jerison, Rings of Continuous Functions (Van Nostrand, Princeton, 1960).

[14] J. Madden and J. Vermeer, 'Lindelöf locales and realcompactness', Math. Proc. Cambridge Philos. Soc. 99 (1986), 473-480.

[15] N. Marcus, 'Realcompactification of frames', Comment. Math. Univ. Carolin. 36(2) (1995), 347-356.

[16] J. Picado and A. Pultr, 'Frames and locales: topology without points', in: Frontiers in Mathematics (Birkhäuser, Basel, 2011).

THEMBA DUBE, Department of Mathematical Sciences, University of South Africa, PO Box 392, 0003 Pretoria, South Africa e-mail: dubeta@unisa.ac.za 